\title{
Scan-Rate-Dependent lon Current Rectification and Rectification Inversion in Charged Conical Nanopores
}

\author{
Dmitry Momotenko and Hubert H. Girault*
}

Laboratoire d'Electrochimie Physique et Analytique, Ecole Polytechnique Fédérale de Lausanne, Station 6, CH-1015 Lausanne, Switzerland

Supporting Information

ABSTRACT: Herein we report a theoretical study of diode-like behavior of negatively charged (e.g., glass or silica) nanopores at different potential scan rates $\left(1-1000 \mathrm{~V} \cdot \mathrm{s}^{-1}\right)$. Finite element simulations were used to determine currentvoltage characteristics of conical nanopores at various electrolyte concentrations. This study demonstrates that significant changes in rectification behavior can be observed at high scan rates because the mass transport of ionic species appears sluggish on the time scale of the voltage scan. In particular, it explains the influence of the potential scan rate on the nanopore rectifying properties in the cases of classical rectification, rectification inversion, and the "transition" rectification domain where the rectification direction in the nanopore could be modulated according to the applied scan rate.

$\mathrm{M}$ odeling of molecular and ionic transport through artificial nanopores is useful for understanding the properties and functions of ionic channels in biological membranes between the cell nucleus and the extracellular environment. ${ }^{1}$ In addition, artificial nanopores and nanopipettes are widely used in electrophysiology, ${ }^{2}$ molecular delivery techniques, ${ }^{3}$ single-molecule detection, ${ }^{4}$ nanoparticle characterization, ${ }^{5}$ and information processing ${ }^{6}$ and as tools for spatially resolved measurements. ${ }^{7,8}$ Therefore, the prediction and control of properties in such nanoscale devices have many applications.

Wei and Bard ${ }^{9}$ in 1997 discovered that nanopipette electrodes with opening sizes comparable to the diffuse layer thickness exhibit diodelike properties, i.e., nonlinear current-voltage behavior called ion current rectification (ICR). An asymmetry of the ion current was found to be strongly dependent on the pore diameter, ${ }^{10}$ surface charge density, ${ }^{11,12}$ electrolyte concentration, ${ }^{9}$ and pore geometry. ${ }^{13,14}$ The key issue for the origin of rectification is the asymmetry of the electrolyte distribution within the pore, caused by a high nonlinearity of electric potential profiles ${ }^{15,16}$ and the presence of a perm-selective barrier, ${ }^{9,14}$ at the tip of the pore.

Quantitative description of ICR in conical nanopores can be modeled by computing ionic fluxes $J_{i}$ taking advantage of molecular dynamics ${ }^{17}$ or using finite element (FEM) simulations for solving continuum-based equations. ${ }^{14,16,18}$ In the latter approach, the system of Nernst-Planck-Poisson (NPP) differential equations is solved for the given geometrical model under proper boundary conditions by discretization of the numerical problem using Petrov-Galerkin or Ritz-Galerkin methods. This approach assumes the calculation of the mass flux for species $i$ using the Nernst-Planck equation (eq 1),

$$
J_{i}=-D_{i} \nabla c_{i}-\frac{z_{i} F}{R T} D_{i} c_{i} \nabla \phi+c_{i} u
$$

while taking into account electrostatic effects of the charged wall using the Poisson equation (eq 2),

$$
\nabla^{2} \phi=-\frac{F}{\varepsilon \varepsilon_{0}} \sum_{i} z_{i} c_{i}
$$

In these equations, $D_{i}, c_{i}$, and $z_{i}$ are the diffusion coefficient, concentration, and charge number of species $i$, respectively, while $F, R, T, \phi, u, \varepsilon$, and $\varepsilon_{0}$ are the Faraday constant, the gas constant, the absolute temperature, the Galvani electric potential, the fluid velocity, the relative permittivity of the medium, and the vacuum permittivity, respectively.

Recently, Guerrette and Zhang ${ }^{19}$ demonstrated that the current response of quartz nanopores with diameters in the range $4-25 \mathrm{~nm}$ is a function of the bias scan rate $(v)$. Experimental current-voltage curves taken at low scan rates of $5 \mathrm{~V} \cdot \mathrm{s}^{-1}$ exhibited a strong current asymmetry associated with a strong rectification effect, while at higher scan rates (e.g., $>200 \mathrm{~V} \cdot \mathrm{s}^{-1}$ ) the response tended to be linear, associated with an Ohmic effect. The effect of the bias scan rate was attributed to the fact that the redistribution of the ionic species in the nanopore could be sluggish in comparison with the fast voltage perturbation. However, no quantitative model was proposed.

In the current study, we used FEM simulations to investigate in detail the mechanism of ICR in conical nanopores at different voltage scan rates. Previously we reported FEM simulations of ICR using the NPP equations under steady-state conditions $\left(\partial J_{i} / \partial t=0\right)$ for conical nanopores of different geometries, ${ }^{14}$ and our results demonstrated the existence of an unusual rectification behavior in diluted electrolytes, namely, rectification inversion. In addition, to the best of our knowledge, no examples showing the theoretical treatment of ICR taking into account time as a variable (i.e., under different voltage scan rates) have been provided. In this work, we solved the above-mentioned system of differential equations in transient form for conical nanopores with a half-cone angle of $5^{\circ}$ carrying negatively charged walls with a surface charge density of $1 \mathrm{mC} \cdot \mathrm{m}^{-2}$, which was shown to be the charge of glass and silica surfaces in diluted electrolytes. ${ }^{20}$ The sign convention used in this paper specifies positive bias as

Received: May 26, 2011

Published: August 18, 2011 

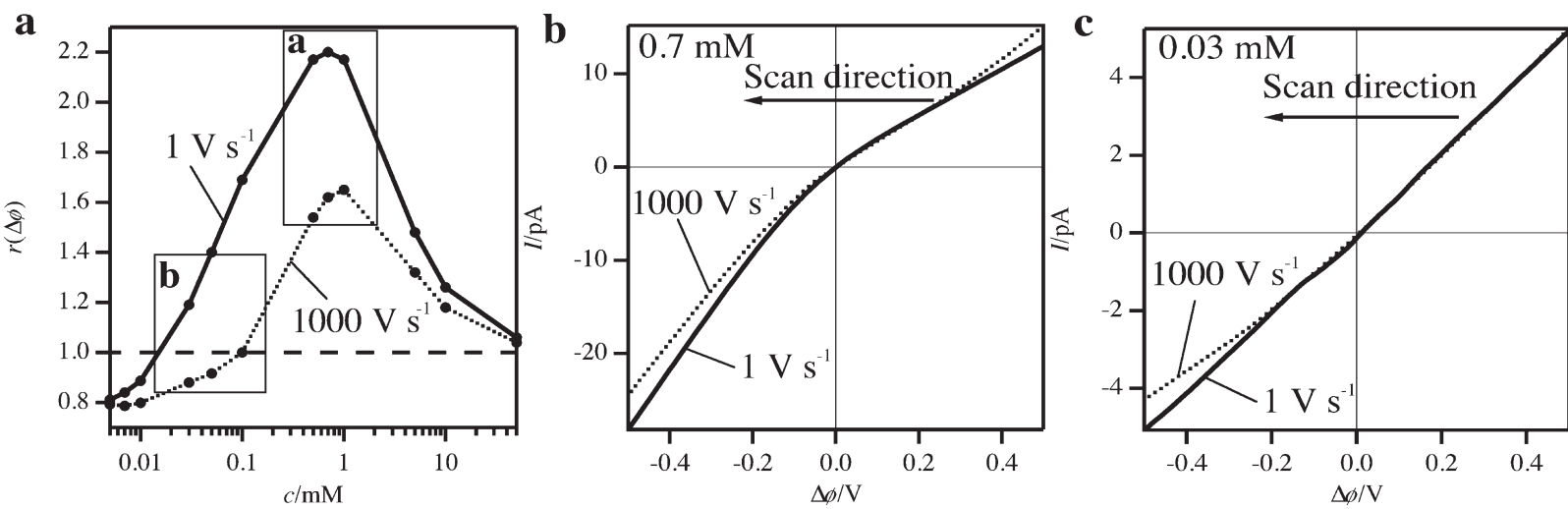

Figure 1. (a) Rectification ratio $r(\Delta \phi)=|I(-0.5 \mathrm{~V}) / I(+0.5 \mathrm{~V})|$ as the function of bulk electrolyte concentration $c$ (given on a logarithmic scale) at scan rates of $1 \mathrm{~V} \cdot \mathrm{s}^{-1}$ (solid line) and $1000 \mathrm{~V} \cdot \mathrm{s}^{-1}$ (dotted line). The dashed line at $r(\Delta \phi)=1$ shows the absence of rectification. (b, c) Corresponding current-voltage characteristics of a conical nanopore at 0.7 and $0.03 \mathrm{mM} \mathrm{KCl}$ (marked as "a" and "b", respectively, in (a)). Arrows on the graphs show the scan direction.

the Galvani potential difference between the equipotential placed closer to the larger pore orifice vs that placed closer to the pore tip. The electrical potential difference was modulated as $\Delta \phi=$ $\Delta \phi_{0}-v t$, where $\Delta \phi_{0}$ specifies the initial Galvani potential difference between the equipotentials, so the applied voltage was scanned from positive to negative values (for more details, see section SI-1 in the Supporting Information).

Figure 1 demonstrates the rectifying properties of a nanopore at different electrolyte concentrations when a bias is applied at scan rates of 1 and $1000 \mathrm{~V} \cdot \mathrm{s}^{-1}$. The rectification effect is characterized in terms of the rectification ratio $r(\Delta \phi)=|I(-\Delta \phi) / I(\Delta \phi)|$, where $I(\Delta \phi)$ and $I(-\Delta \phi)$ specify the currents at positive and negative biases, respectively (Figure 1a). As expected, at low scan rates (e.g., $\left.1 \mathrm{~V} \cdot \mathrm{s}^{-1}\right)$, the rectification behavior of the nanopore is very close to the steady-state response (see section SI-2), and the pore exhibits classical rectifying properties. Under such conditions, the rectification effect shows a maximum ratio $r(\Delta \phi)=2.2$ at a $\mathrm{KCl}$ concentration of $0.7 \mathrm{mM}$, and at lower electrolyte concentrations $(<0.02 \mathrm{mM})$, rectification inversion is observed. When fast voltage variations (i.e., $1000 \mathrm{~V} \cdot \mathrm{s}^{-1}$ ) are applied, the rectification ratios diminish significantly for almost all concentrations, with a maximum $r(\Delta \phi)$ value of only 1.65 at $1 \mathrm{mM} \mathrm{KCl}$. The ICR inversion appears at ionic strengths that are higher by at least 1 order of magnitude than in steady-state case.

The rectifying characteristics of the nanopore at different electrolyte conditions can be divided into two regions (marked as "a" and "b" in Figure 1a). In the classical rectification domain $[r(\Delta \phi)>1$, region a in Figure 1a], the rectification ratio tends to its maximum value, and the ICR inversion is not observed in the scan rate range $1-1000 \mathrm{~V} \cdot \mathrm{s}^{-1}$. The corresponding current-voltage curves in Figure $1 \mathrm{~b}$ demonstrate trends similar to those reported in the experimental work of Guerrette and Zhang ${ }^{19}$ (e.g., the straightening of the diodelike characteristics at high scan rates), while at steady state $\left(1 \mathrm{~V} \cdot \mathrm{s}^{-1}\right)$, typical nonlinear responses are observed.

Within the other concentration domain (Figure 1a, region b) the nanopore rectifies the ionic current in both directions. Varying the scan rate, one can obtain both type of currentvoltage characteristics of a nanopore, namely the classical rectification $(r(\Delta \phi)>1$; Figure 1c, solid line $)$ and the rectification in version $(r(\Delta \phi)<1$; Figure 1c, dotted line).

Figure 2 shows the influence of the voltage scan rate on the rectification ratio at different electrolyte concentrations.
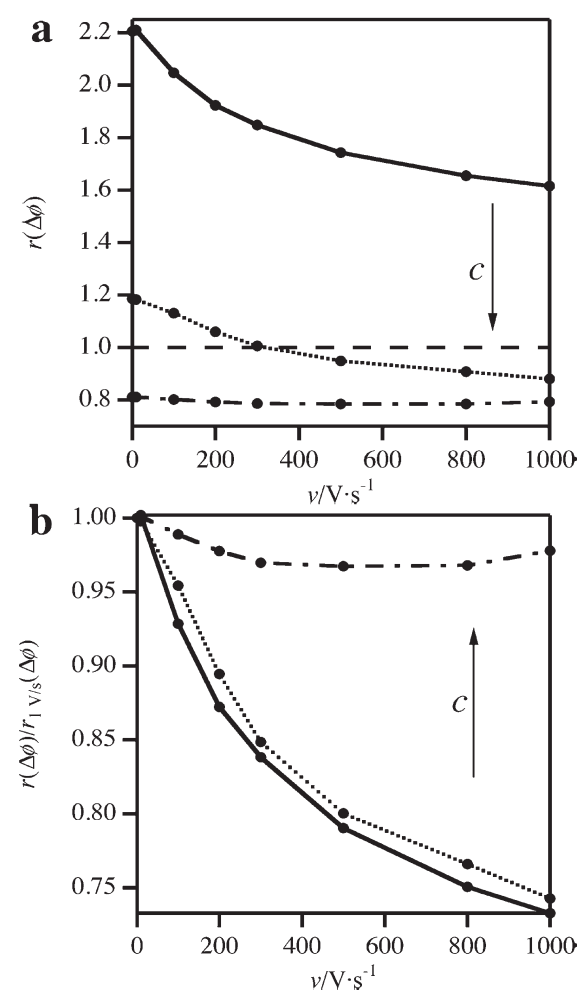

Figure 2. Effect of the voltage scan rate $(v)$ on the magnitude of ICR at different electrolyte concentrations $(0.7 \mathrm{mM}, 0.03 \mathrm{mM}$, and $5 \mu \mathrm{M}$ for solid, dotted, and dash-dotted lines, respectively). The effect is represented using (a) the absolute value of the rectification ratio and (b) the relative value $r(\Delta \phi) / r_{1 \mathrm{~V} / \mathrm{s}}(\Delta \phi)$, i.e., the rectification ratio normalized to its value under steady-state conditions $\left(v=1 \mathrm{~V} \cdot \mathrm{s}^{-1}\right)$. Arrows on the graphs indicate increasing bulk electrolyte concentration. In (a), the dashed line $r(\Delta \phi)=1$ shows the absence of rectification.

The general trend is a decrease in the rectification ratio with increasing scan rate that is in agreement with the previously reported experimental results. ${ }^{19}$ Interestingly, the relative magnitude of the scan rate effect (Figure $2 b$ ) is very similar at both classical and "transition" rectification domains (Figure 1a, region $b$ ), and only at low electrolyte concentrations (e.g., $5 \mu \mathrm{M}$ ) the effect 

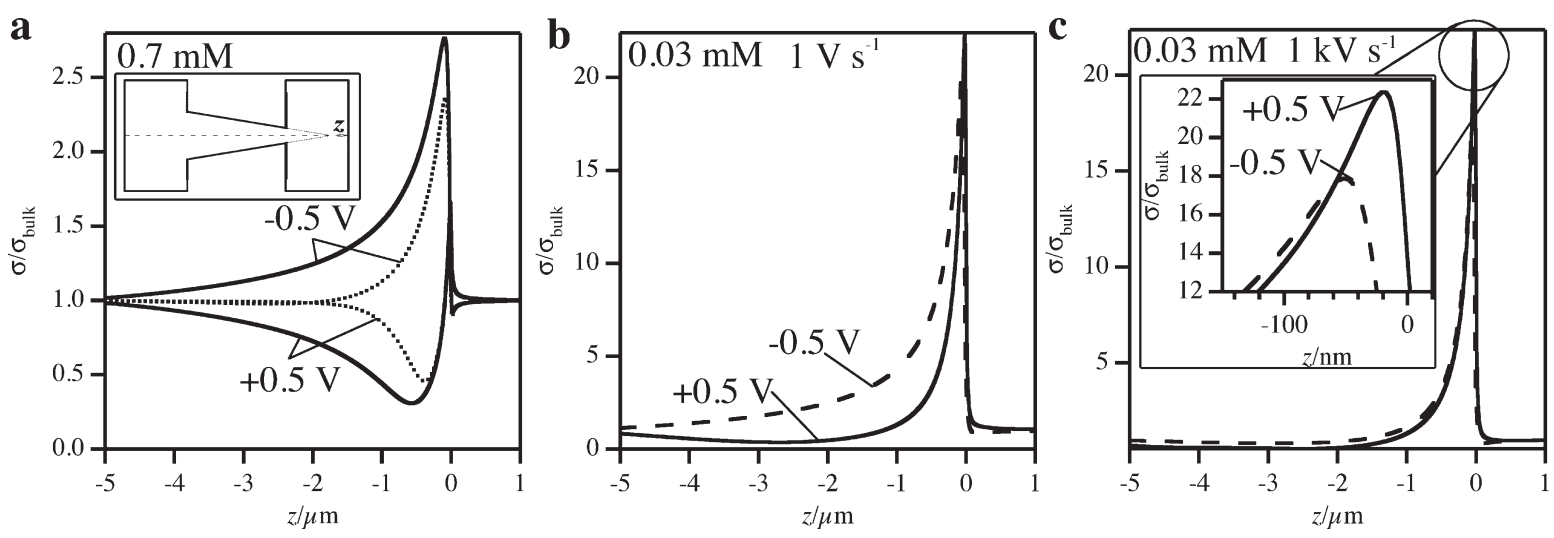

Figure 3. (a) Relative ionic conductivity distributions $\sigma / \sigma_{\text {bulk }}$ (normalized to the bulk value) along the $z$ axis of the conical nanopore (schematically represented in the inset) at scan rates of $1 \mathrm{~V} \cdot \mathrm{s}^{-1}$ (solid line) and $1000 \mathrm{~V} \cdot \mathrm{s}^{-1}$ (dotted line) in the classical rectification domain $(0.7 \mathrm{mM} \mathrm{KCl})$. (b, c) Distributions of $\sigma / \sigma_{\text {bulk }}$ in the "transition" concentration domain $(0.03 \mathrm{mM})$ at scan rates of (b) $1 \mathrm{~V} \cdot \mathrm{s}^{-1}$ and $(\mathrm{c}) 1000 \mathrm{~V} \cdot \mathrm{s}^{-1}$ at bias values $\Delta \phi$ of $+0.5 \mathrm{~V}$ (solid lines) and $-0.5 \mathrm{~V}$ (dashed lines). The inset in (c) shows a magnified view in the tip region of the nanopore.

of the scan rate on ICR is almost negligible. At very high scan rates (e.g., $1000 \mathrm{~V} \cdot \mathrm{s}^{-1}$ ), the direction of the potential scan has an influence on the rectification factor and the shape of the corresponding current-voltage characteristics of the nanopore (see section SI-3). Despite, qualitatively computational results are similar to those reported by Guerrette et al. ${ }^{19}$ the magnitude of this effect is relatively small compared to the experimental results. The influence of the scan direction is stronger in the classical rectification domain, whereas the rectifying properties of the nanopore in the "transition" concentration range remain almost independent of the sign of the initial bias.

In order to describe the mechanisms driving rectification at high scan rates, it is worthwhile first to refer to the ICR origin under steady-state (or low scan rate) conditions. It was shown previously ${ }^{14,16}$ that rectification is governed mainly by conductivity changes near the tip of the nanopore. In the case of classical rectification, the fact that the mass-transport rate is lower inside than outside the pore coupled with the presence of a permselective region at the tip results in ionic depletion at positive potentials and enrichment at negative ones. ${ }^{14,16}$ At very low ionic strengths, rectification inversion can be encountered because of the significant conductivity changes inside the perm-selective plug due to the strong Gouy-Chapman layer interaction in the tip region. The aforementioned behavior was demonstrated previously with the use of an analytical model and numerical computations. ${ }^{14}$

The present simulations of ICR at high scan rates show that rectification is influenced by the rate of ionic redistribution inside the pore, as was proposed by Guerrette and Zhang. ${ }^{19}$ According to the computational results at high scan rates, the diffusion contribution to the total flux remains almost constant on the time scale of the voltage scan, whereas the migration component of the current leads to rectification (see section SI-4). In other words, at high scan rates, diffusion is delayed in comparison with the steady-state case, causing a decrease in perm-selectivity at the pore tip. At low scan rates (e.g., $1 \mathrm{~V} \cdot \mathrm{s}^{-1}$ ) in the classical rectification domain $(0.7 \mathrm{mM})$, most of the current within the nanopore is transported by cations (e.g., the transport number of $\mathrm{K}^{+}$at a bias equal to $0.5 \mathrm{~V}$ is $t_{+}=0.79$, while this value drops to 0.53 at $\left.v=1000 \mathrm{~V} \cdot \mathrm{s}^{-1}\right)$. As shown previously, the permselectivity of the pore is a key issue for ICR in charged conical nanopores, ${ }^{9,14}$ and as the loss of perm-selective properties occurs, the rectification diminishes, resulting in a nearly Ohmic behavior at $1000 \mathrm{~V} \cdot \mathrm{s}^{-1}$. The decrease in perm-selectivity can be also illustrated (see Figure 3a) as less effective depletion and enrichment of the pore conductivity at positive and negative biases, respectively. In other words, when a fast voltage perturbation is applied (e.g., $1000 \mathrm{~V} / \mathrm{s}$ ), the delayed ionic transport rate leads to a decrease in ionic enrichment/depletion regions, resulting in loss of rectification.

In more dilute electrolytes, an accumulation of ionic species also occurs inside the perm-selective plug just at the very tip of the pore, as shown in Figure 3b. Under steady-state conditions, this leads to a decrease in the rectification and can even lead to rectification inversion. A comparison of the ionic distributions at $1 \mathrm{~V} \cdot \mathrm{s}^{-1}$ (Figure $3 \mathrm{~b}$ ) and $1000 \mathrm{~V} \cdot \mathrm{s}^{-1}$ (Figure 3c) for the "transition" rectification domain shows the effect of delayed ionic transport at high voltage scan rates. At high scan rates, ionic redistribution appears sluggish in comparison with the rapidly changing voltage, and the conductivity changes occur mainly within the perm-selective region of the nanopore (i.e., closer to the tip of the nanopore). Consequently, the ICR behavior changes from classical rectification at $1 \mathrm{~V} \cdot \mathrm{s}^{-1}$, where depletion/ enrichment of ionic species occurs within the inner space of the pore, to rectification inversion at $1000 \mathrm{~V} \cdot \mathrm{s}^{-1}$, where the conductivity variation takes place only at the very tip. At very low electrolyte concentrations (e.g., $5 \mu \mathrm{M}$ ), the influence of the scan rate is insignificant, probably because of the very strong permselectivity of the pore tip.

In summary, ion current rectification at different voltage scan rates in charged conical nanopores depends on the relatively sluggish ion redistribution controlling the perm-selective properties of the nanopore. At high scan rates, the perm-selectivity of the pore in the classical rectification domain is scarcely established, and the nanopore behaves as an Ohmic resistor. In addition, we report the existence of a "transition" rectification domain, i.e., a concentration domain where nanopores can rectify ionic current in both directions according to the scan rate applied. The presented transient calculations complement and contribute as additional evidence for rectification inversion in charged conical nanopores under low ionic strength conditions. This diode-like behavior can find important applications, as the rectifying properties of the nanopore could be tuned using 
appropriate potential scan rates. This unusual frequency-dependent behavior has no reported analogues for typical diodes based on $\mathrm{p}-\mathrm{n}$ junctions.

\section{ASSOCIATED CONTENT}

S Supporting Information. Details of the FEM simulations procedure, steady-state rectification data (and the validation for the transient computational model), influence of the scan direction on the current-voltage characteristics of the pore, and computed migration and diffusion components of the total ionic current at low and high potential scan rates. This material is available free of charge via the Internet at http://pubs.acs.org.

\section{AUTHOR INFORMATION}

\section{Corresponding Author}

hubert.girault@epfl.ch

\section{ACKNOWLEDGMENT}

This work was supported by SNCF Grants 20735001 and 20628506 and by SNF Grant 20PA21_121570/1 "HighThroughput SECM Imaging”. The authors also acknowledge Jacques Josserand for assistance with the FEM simulations.

\section{REFERENCES}

(1) Hille, B. Ionic Channels of Excitable Membranes, 3rd ed.; Sinauer Associates: Sunderland, MA, 1992.

(2) Korchev, Y. E.; Negulyaev, Y. A.; Edwards, C. R. W.; Vodyanoy, I.; Lab, M. J. Nat. Cell Biol. 2000, 2, 616.

(3) Ying, L. M. Biochem. Soc. Trans. 2009, 37, 702.

(4) Harrell, C. C.; Choi, Y.; Horne, L. P.; Baker, L. A.; Siwy, Z. S.; Martin, C. R. Langmuir 2006, 22, 10837.

(5) Lan, W.-J.; Holden, D. A.; Zhang, B.; White, H. S. Anal. Chem. 2011, 83, 3840.

(6) Cervera, J.; Ramirez, P.; Mafe, S. Electrochim. Acta 2011, 56, 4504.

(7) Hansma, P. K.; Drake, B.; Marti, O.; Gould, S. A. C.; Prater, C. B. Science 1989, 243, 641.

(8) Elsamadisi, P.; Wang, Y. X.; Velmurugan, J.; Mirkin, M. V. Anal. Chem. 2011, 83, 671.

(9) Wei, C.; Bard, A. J.; Feldberg, S. W. Anal. Chem. 1997, 69, 4627.

(10) Kovarik, M. L.; Zhou, K. M.; Jacobson, S. C. J. Phys. Chem. B 2009, 113, 15960.

(11) Siwy, Z.; Heins, E.; Harrell, C. C.; Kohli, P.; Martin, C. R. J. Am. Chem. Soc. 2004, 126, 10850.

(12) Wang, X. W.; Xue, J. M.; Wang, L.; Guo, W.; Zhang, W. M.; Wang, Y. G.; Liu, Q.; Ji, H.; Ouyang, Q. Y. J. Phys. D: Appl. Phys. 2007, 40, 7077 .

(13) Ramírez, P.; Apel, P. Y.; Cervera, J.; Mafé, S. Nanotechnology 2008, 19, No. 315707.

(14) Momotenko, D.; Cortés-Salazar, F.; Josserand, J.; Liu, S.; Shao, Y.; Girault, H. H. Phys. Chem. Chem. Phys. 2011, 13, 5430.

(15) Siwy, Z.; Fulinski, A. Am. J. Phys. 2004, 72, 567.

(16) White, H. S.; Bund, A. Langmuir 2008, 24, 2212.

(17) Xue, J. M.; Zou, X. Q.; Xie, Y. B.; Wang, Y. G. J. Phys. D: Appl. Phys. 2009, 42, No. 105308.

(18) Liu, Q.; Wang, Y.; Guo, W.; Ji, H.; Xue, J.; Ouyang, Q. Phys. Rev. E 2007, 75, No. 051201.

(19) Guerrette, J. P.; Zhang, B. J. Am. Chem. Soc. 2010, 132, 17088.

(20) Behrens, S. H.; Grier, D. G. J. Chem. Phys. 2001, 115, 6716. 